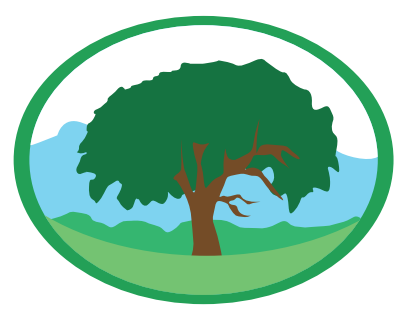

\title{
ESTUDO DA OBTENCÃO DE NANOPARTÍCULAS DE TIO2 PELOS MÉTODOS SOLVOTÉRMIICO ASSISTIDO POR MICRO-ONDAS E SOL-GEL
}

\author{
SEIXAS, J. N. ${ }^{1}$; SILVA, G. E. H. ${ }^{1}$; NASCIMENTO, L. E. ${ }^{2}$; NETRO, N. F. A. ${ }^{2}$; MOTTA, F. V. ${ }^{2}$; CARREÑO, N. L. V. ${ }^{1}$. \\ 1 UFPEL- Universidade Federal de Pelotas. \\ ${ }^{2}$ UFRN- Universidade Federal do Rio Grande do Norte.
}

Palavras-chave: dióxido de titânio, sol-gel, síntese solvotérmica assistida por micro-ondas.

\section{Resumo}

Nanopartículas de dióxido de titânio $\left(\mathrm{TiO}_{2}\right)$ possuem elevado potencial para aplicação ambiental em função de suas propriedades fotocatalíticas. Entretanto, para otimizar tais propriedades, o tamanho de partícula, estrutura, fase e área superficial são parâmetros que necessitam de especial atenção durante a síntese. A utilização de diferentes métodos de obtenção possibilita o controle destas características. Nesse trabalho, pós de $\mathrm{TiO}_{2}$ foram obtidos pelos métodos solvotérmico assistida por micro-ondas e sol-gel para avaliação das cristalinidades, fases e morfologias obtidas. Os difratogramas indicam que a fase anatase foi obtida para ambas as metodologias, com a cristalinidade sendo inferior para a metodologia solvotérmica e para a metodologia sol-gel quando não foi realizada a calcinação. Além disso, na metodologia sol-gel, para maiores temperaturas de calcinação, ocorre menor formação de agregados, adicionalmente foi possível observar a formação de anéis de $\mathrm{TiO}_{2}$, ainda que com presença irregular.

\section{STUDY OF THE OBTAINING OF TIO2 NANOPARTICLES BY THE SOLVOTHERMIC METHODS ASSISTED BY MICROWAVE AND SOL-GEL}

Keywords: titanium dioxide, sol-gel, microwave-assisted solvothermic synthesis

\begin{abstract}
Nanoparticles of titanium oxide $\left(\mathrm{TiO}_{2}\right)$ have high potential for environmental application due to their photocatalytic properties. However, to optimize such properties, particle size, structure, phase and surface area are parameters that need attention. The use of different synthesis methods makes possible to control these characteristics. In this work, $\mathrm{TiO}_{2}$ powders were obtained by the microwave assisted solder gel and sol-gel methods to evaluate the crystallinities, phases and morphologies obtained. The diffractograms indicate that the anatase phase was obtained for both methodologies, with the crystallinity being lower for the solvothermic methodology and for the sol-gel methodology when the calcination was not performed. Furthermore, in the sol-gel methodology, for higher calcination temperatures, less formation of aggregates occurs, in addition it was possible to observe the formation of $\mathrm{TiO}_{2}$ rings, although with irregular presence.
\end{abstract}




\section{INTRODUÇÃO}

O avanço industrial é um dos fatores que intensificaram os efeitos da atividade humana no meio ambiente. A consequência disso é uma condição de poluição da água, do solo e do ar e de quase esgotamento dos recursos naturais do planeta (HAIDER et al., 2017). Numa tentativa de remediar esses problemas, diversos pesquisadores vêm estudando o $\mathrm{TiO}_{2}$ como uma alternativa, com base nas suas propriedades de fotocatalisador, capacidade de absorção no UV, transparência no visível e elevado índice de refração (DEVICALA et al., 2018). Essas características tornam o $\mathrm{TiO}_{2}$ um material com alto potencial de aplicação ambiental pois o torna interessante para reaçôes fotocatalíticas, como conversão de $\mathrm{CO}_{2}$, processos de oxidação avançada (POA), tratamento de água, controle de qualidade do ar e armazenamento de energia. Notoriamente, para muitas destas variadas aplicaçóes, o tamanho de partícula, estrutura cristalina e fase, porosidade e área superficial influenciam a atividade do $\mathrm{TiO}_{2}$ dramaticamente ( $\mathrm{ZU}$ et al., 2018). Da mesma forma, a obtenção de diferentes morfologias é uma consequência direta do tipo de síntese utilizada (WANG et al., 2014). A técnica sol-gel tem sido bastante utilizada pois apresenta vantagens como possibilidade de síntese em baixas temperaturas, variedade de precursores, e simplicidade de rota (CASINO et al., 2014). Entretanto, a metodologia sol-gel baseada em uma etapa de solubilização de alcóxidos pode apresentar dificuldades no controle de estrutura obtida assim como também pode gerar partículas amorfas (WANG et al., 2014).

Por sua vez, a síntese solvotérmica assistida por micro-ondas tem sido amplamente utilizada na obtenção de diferentes materiais, incluindo o $\mathrm{TiO}_{2}$. Esse método possui um aquecimento rápido $\mathrm{e}$ uniforme característicos da penetração da radiação das micro-ondas (ANDRADE NETO et al., 2018) e uma cinética rápida de cristalização (MAHMOUDI; BOUDJADAR, 2013). Consequentemente, apresenta maior controle no tamanho das partículas geradas e pode gerar partículas com menores aglomeraçóes e maior cristalinidade quando comparado a outras metodologias.

Considerando que ambas metodologias são utilizadas na síntese do $\mathrm{TiO}_{2}$, o objetivo do presente trabalho foi avaliar o efeito da utilização dessas duas metodologias, a síntese solvotérmica assistida por micro-ondas e a sol-gel, na morfologia do $\mathrm{TiO}_{2}$, comparando diferentes temperaturas de síntese para cada metodologia.

\section{MATERIAL E MÉTODOS}

\subsection{Método solvotérmico assistido por micro- ondas}

A síntese seguiu uma adaptação de uma metodologia conhecida na literatura (FAN et al., 2016). Para tanto, diluiu-se $10 \mathrm{ml}$ de isopropóxido de titânio em $30 \mathrm{ml}$ etanol, o sistema foi mantido sob agitação constante por $5 \mathrm{~min}$, seguiu-se da adição gota-a-gota de $20 \mathrm{ml}$ de ácido acético com posterior adição de $1 \mathrm{ml}$ ácido sulfúrico. Manteve-se a agitação por 20 min e então a solução foi sonicada por $1 \mathrm{~h}$ à $60^{\circ} \mathrm{C}$. $\mathrm{O}$ material obtido foi levado a uma autoclave revestida por teflon para o tratamento térmico por irradiação micro-ondas na temperatura de $120{ }^{\circ} \mathrm{C}$ durante $20 \mathrm{~min}$. Após o resfriamento, o precipitado foi centrifugado e lavado com água destilada e etanol até $\mathrm{pH}$ neutro, então foi levado para secagem na estufa e macerado até a obtenção de um pó.

\subsection{Método sol-gel}

Nessa síntese, $\mathrm{o} \mathrm{TiO}_{2}$ foi obtido por uma adaptação de uma metodologia conhecida pela literatura (BEHNAJADY et al., 2011). Realizou-se a diluição de 18,6 mL de isopróxido de titânio em 35,8 mL em ácido acético glacial, sob banho térmico de $0{ }^{\circ} \mathrm{C}$. Adicionou-se gota-a-gota $395 \mathrm{~mL}$ de água destilada sob agitação por 1 hora com posterior sonicação em um ultrassom de banho por 30 minutos. O material obtido foi mantido numa caixa no escuro por 12 horas e então levado à estufa a $80^{\circ} \mathrm{C}$ até nucleação e completa secagem para posteriormente ser calcinado. Foram usadas as temperaturas de $450{ }^{\circ} \mathrm{C}$ e $550{ }^{\circ} \mathrm{C}$ para calcinação durante $3 \mathrm{~h}$. $\mathrm{O}$ material obtido foi macerado até a obtenção de um pó.

\subsection{Caracterizaçáo}

A análise de difração de raios-X (DRX) foi utilizada para identificação das fases e quantificação da cristalinidade dos pós de $\mathrm{TiO}_{2}$, com os parâmetros 
de voltagem de análise de $40 \mathrm{kV}$, ângulo de varredura de $5^{\circ}-80^{\circ}$ e velocidade de $1 \% / m i n$ no equipamento Shimadzu, modelo XRD-7000. Além disso, observou-se morfologia por microscopia eletrônica de varredura por emissão de campo (MEV-FEG) em um equipamento da marca Zeiss, modelo ZEISS Sigma 300. O preparo da amostra seguiu a solubilização dos pós em um solvente (acetona) para posterior gotejamento $(15 \mu \mathrm{l})$ numa fita de carbono colada no porta-amostra, seguiu-se a secagem das amostras e então as análises foram realizadas.

\section{RESULTADOS E DISCUSSÃO}

\subsection{Cristalinidade}

Na Figura 1 sáo apresentados os resultados de DRX para as amostras obtidas pela metodologia sol-gel e solvotérmica.
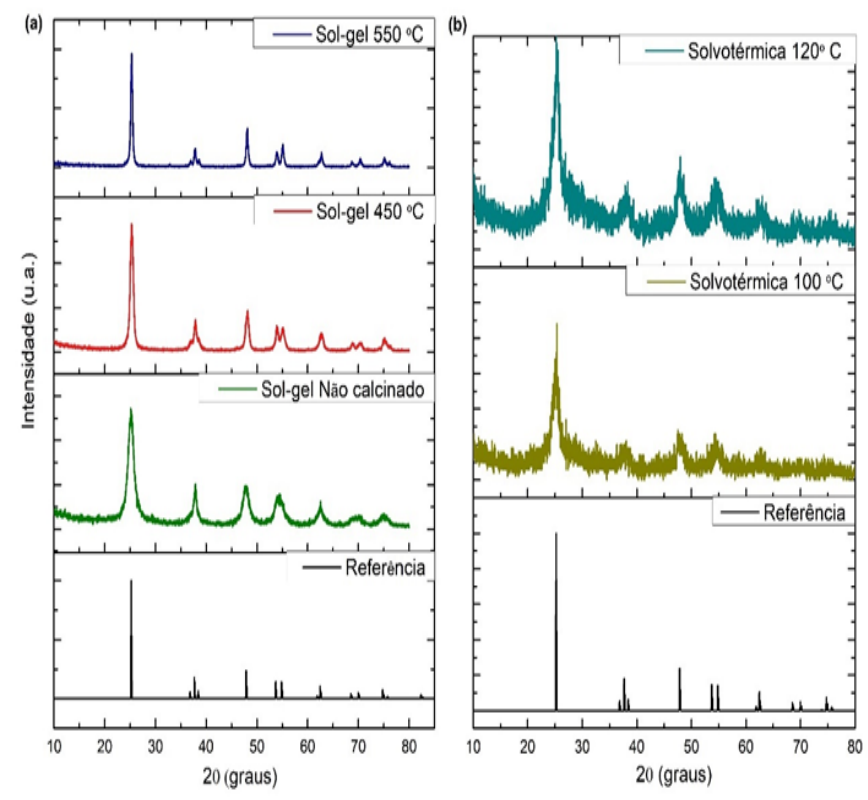

Figura 1. (a) Difratogramas das amostras obtidas por sol-gel sem calcinação e com diferentes temperaturas de calcinação (b) Difratogramas das amostras obtidas por síntese solvotérmica em diferentes temperaturas.

Percebe-se pelos difratogramas de raios-X, a presença de picos nos ângulos $2 \theta$ iguais a $25,40^{\circ}, 38,28^{\circ}, 47,46^{\circ}$, $54,26^{\circ}$ e $62,68^{\circ}$, atribuídos aos planos de difração (101), (004), (200), (105) e (204), respectivamente (YANG et al., 2017). Esse perfil cristalográfico é referente à fase anatase do $\mathrm{TiO}_{2}$ identificado pela ficha cristalográfica no 7206075 do COD (Crystallography Open Database), utilizado como referência. Deve-se destacar que em todas as sínteses foi possível obter majoritariamente a fase anatase. Entretanto, para uma interpretação mais correta dos difratogramas é preciso considerar que o plano cristalográfico (101) do pico de difração da fase anatase se sobrepõe aos planos cristalográficos (120) e (111) da fase bruquita em $20=25,34^{\circ}$ e $25,69^{\circ}$, respectivamente (DI PAOLA; BELLARDITA; PALMISANO, 2013). Fora essa possível sobreposiçáo, não se observa nas amostras obtidas nenhum outro indício da presença de outra fase além da anatase. Sabe-se que a fase rutilo é a fase mais estável dos alótropos do $\mathrm{TiO}_{2}$ sendo que a fase bruquita e a fase anatase são consideradas metaestáveis e podem se transformar na fase rutilo quando tratadas em altas temperaturas (BESSEKHOUAD; ROBERT; WEBER, 2003). Tanto a fase anatase quanto a rutilo apresentam atividade fotocatalítica de interesse. Apenas recentemente foi possível definir uma síntese que permitisse a obtenção da fase bruquita pura e pode-se avaliar que essa fase possui baixa atividade fotocatalítica.

A larga utilização da metodologia sol-gel para obtenção de $\mathrm{TiO}_{2}$ se justifica pelas vantagens de possibilidade de síntese que gera materiais com boa cristalinidade, apresenta a possibilidade de utilização de uma variedade de precursores e baixo preço uma vez que náo depende de equipamentos de elevado custo e a temperatura de utilização pode ser baixa. Dependendo do tipo de precursor utilizado, duas abordagens diferentes da síntese sol-gel são definidas: uma onde o processo é baseado em álcool e o precursor de titânio é um alcóxido de metal e outra onde o precursor é um sal de metal inorgânico.

$\mathrm{Na}$ presença de água, os alcóxidos, como o isopropóxido de titânio, são hidrolisados e posteriormente polimerizados para formar uma rede de óxido tridimensional. Descrevendo mais detalhadamente as etapas envolvidas, pode-se dividir a obtenção de pós de $\mathrm{TiO}_{2}$ pela metodologia sol-gel utilizando alcóxidos em 4 etapas, são elas: a hidrólise, a condensaçáo, a secagem e a calcinaçáo. Como resultado essas reações formam colóides dos quais pode-se obter pós. Esses pós terão morfologia e cristalinidade definidas pelas condiçóes experimentais. As reaçóes de hidrólise e condensação estão esquematizadas de acordo com as equaçóes abaixo: 


$$
\begin{array}{cc}
\mathrm{Ti}(\mathrm{OR})_{4}+4 \mathrm{H}_{2} \mathrm{O} \rightarrow \mathrm{Ti}(\mathrm{OH})_{4}+4 \mathrm{ROH} & \text { Equação (1) } \\
\mathrm{Ti}(\mathrm{OH})_{4} \rightarrow \mathrm{TiO}_{2}+\mathrm{H}_{2} \mathrm{O} & \text { Equação (2) }
\end{array}
$$

Sendo a Eq. 1 a reação de hidrólise e a Eq. 2 de condensação, onde $\mathrm{R}$ pode ser um radical etilo, i-propilo, n-butilo, etc. (CHIMIE et al., 1989). A etapa de condensaçáo ocorre simultaneamente de duas formas, via olação, ou seja, com a formação de grupos hidroxos entre os metais, ou via oxolação, ou seja, com a formação de ligaçôes oxo entre dois cátions metálicos. O processo de oxolaçáo, por sua vez, pode ocorrer por adição nucleoflílica, quando a esfera de coordenação do metal não está completamente saturada, ou ainda por substituição nucleofílica, quando a esfera de coordenação do metal está saturada (GONÇALVES, 2011).

Quando ocorrer desoxolação, que é a reação inversa a oxolação, Eq. 3, antes da olação, gera-se um crescimento linear ao longo de uma das duas direçôes equivalentes do plano equatorial dos dímeros. Então, essas cadeias lineares de $\mathrm{TiO}(\mathrm{OH})_{2}(\mathrm{OH})_{2}$ obtidas pela oxolação, sofrem uma transferência interna de um próton. Essa transferência leva a formação de cadeias octaédricas com compartilhamento (pontes de $\mathrm{Ti}_{3} \mathrm{O}$ ) características da fase rutilo. A formação do rutilo pode então estar associado à metaestabilidade das ligaçóes duplas de $\mathrm{Ti}$ e $\mathrm{Ti}$, entre monômeros e dimêros. Se a desoxolação ocorrer antes da olação, a condensação pode avançar ao longo da direção apical para cadeias distorcidas típicas da estrutura do tipo anatase. Esse controle da desoxolação antes da olação pode ser obtido ajustando o pH e a concentração inicial da água (MAHSHID, 2007).

$$
\mathrm{O}=\mathrm{TiOH}_{2} \rightarrow \mathrm{HO}-\mathrm{Ti}-\mathrm{Ti}-\mathrm{OH}
$$

Eqquação (3)

A fase anatase do $\mathrm{TiO}_{2}$ geralmente é preferida em função de sua alta atividade fotocatalítica, pois tem energia potencial do band gap de condução menor, elevada área específica, não toxicidade, estabilidade fotoquímica e custo barato (MACWAN; DAVE; CHATURVEDI, 2011). O material obtido após a condensação é um gel, que passará pelo processo de secagem para remoção do solvente. Para a próxima etapa, a calcinação, é necessária a utilização de altas temperaturas para a remoçáo do precursor orgânico (GUPTA; TRIPATHI, 2012).
Dessa forma, para a metodologia sol-gel utilizada, há indícios que na reação de condensação tenha ocorrido desoxolação antes do processo de olação. Essa condição pode ter sido alcançada em função do meio ácido utilizado sugerindo ser eficiente para garantir a formação da fase anatase.

O material não calcinado se apresentou menos cristalino, característica que vai se perdendo com o aumento da temperatura resultando na formaçáo de picos bastante definidos que indicam alta cristalinidade. Esse resultado está de acordo com o encontrado na literatura onde está estabelecido que a transformação do $\mathrm{TiO}_{2}$ durante a síntese sol-gel da fase amorfa para a fase anatase é completada na faixa de temperatura de 300 a $600{ }^{\circ} \mathrm{C}$, já a transformação da fase anatase para a fase rutilo ocorre em temperaturas mais altas, na faixa de 400 a $1200{ }^{\circ} \mathrm{C}$, dependendo das condiçóes de preparo de síntese (HANAOR; SORRELL, 2011). Assim, para aplicaçóes onde elevada cristalinidade da fase anatase não são necessárias é possível utilizar o material sem calcinação.

Pode-se descrever a síntese solvotérmica como uma reação ou uma transformação em um meio de solvente orgânico sob efeito de temperatura e pressão. Os mecanismos que agem durante a síntese hidrotermal se repetem na síntese solvotérmica, desta forma, pode-se fazer analogias entre resultados obtidos com as duas metodologias. O principal processo envolvido na síntese hidrotérmica do $\mathrm{TiO}_{2}$ é denominado dissolução-precipitação, considerando que a síntese solvotérmica utililiza solventes orgânicos que por sua vez apresentam constantes dielétricas baixas que resultam numa menor solubulidade do $\mathrm{TiO}_{2}$ e por consequência menor desidratação. Essa menor desidratação gera as partículas obtidas pela síntese solvotérmica partículas menores e com melhor cristalinidade quando comparadas às partículas obtidas pela síntese hidrotérmica (NAM; YANG; DUC, 2013). Novamente, da mesma forma que na síntese hidrotérmica, para a síntese solvotérmica, os produtos obtidos irão depender principalmente dos solventes, da temperatura de reaçáo, do $\mathrm{pH}$ e do tempo de calcinação quando utilizado (WANG et al., 2014).

Quando essa metodologia foi utilizada para a obtenção de $\mathrm{TiO}_{2}$ conforme observa-se no difratograma 
da Figura 1(b) ocorreu a formação exclusivamente da fase anatase para ambas as temperaturas. Para compostos tratados termicamente por esse método, é de conhecimento da literatura que a largura a meia altura dos picos pode ser maior devido a formação de materiais na escala nanométrica (KIM; KWAK, 2007), já a definição dos picos de difração está relacionada à cristalinidade do material e ao tamanho de cristalito. Dessa forma, no caso dos materiais obtidos por síntese

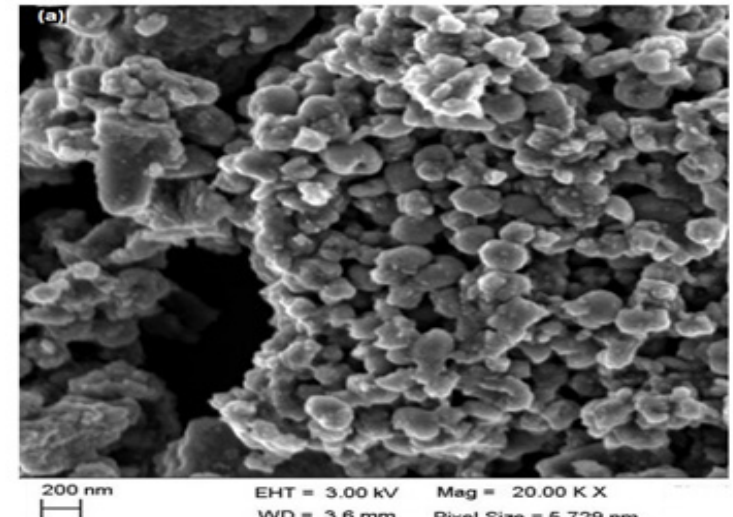

Figura 2. Micrografias de MEV-FEG (a) amostras

Observa-se a formação de diferentes aglomerados de tamanhos variados constituídos de estruturas esféricas na ordem de centenas de nanômetros. Da mesma forma que para metodologia sol-gel, pela síntese solvotérmica é possível obter uma gama variada de morfologias como nanopartículas (SUPPHASRIRONGJAROEN et al., 2008), solvotérmica na temperatura de $120^{\circ} \mathrm{C}$, o alargamento observado para os picos pode indicar uma diminuição do tamanho de cristalito. Além disso, é possível que se otimize a cristalinidade do material com uma posterior calcinação como utilizado na metodologia sol-gel.

\subsection{Morfologia}

$\mathrm{Na}$ Figura 2 são apresentadas as micrografias da amostra obtida por síntese solvotérmica em $100{ }^{\circ} \mathrm{C}$.

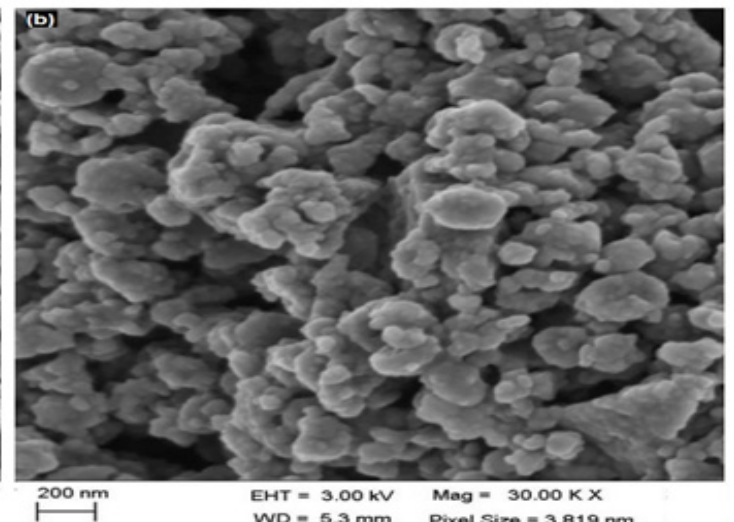

obtidas pela metodologia solvotérmica em $100^{\circ} \mathrm{C}$.

nanotubos (BIAN et al., 2010), esferas vazadas e preenchidas (WANG et al., 2011), nanofolhas, entre outros. No presente trabalho a morfologia obtida foi de esferas de tamanhos variados.

$\mathrm{Na}$ Figura 3 são apresentadas as micrografias dos pós de $\mathrm{TiO}_{2}$ obtidos pela síntese sol-gel.

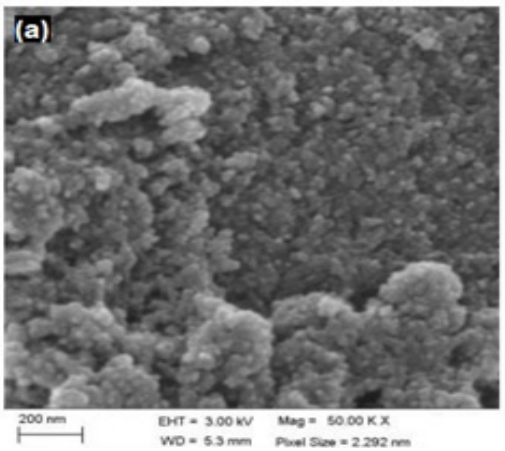

Figura 3. Micrografias de MEV-FEG amostras obtidas pela metodologia: (a) Sol-gel não calcinada; (b) Sol-gel calcinada em $450^{\circ} \mathrm{C}$ e (c) Sol-gel Calcinada em $550^{\circ} \mathrm{C}$

Sabe-se que a metodologia sol-gel pode gerar uma grande variedade de morfologias e de tamanho de partícula, especialmente para o material não calcinado. Essa condição se dá devido ao fato dessa síntese ter rápidas reações de hidrólise e condensação (CHOI; STATHATOS; DIONYSIOU, 2006). Para essa metodologia já foram relatadas a obtenção de morfologias como filmes (ARCONADA et al., 2009; HOU et al., 2009), fibras (YOU et al., 2012), nanopartículas (LÓPEZ; GÓMEZ; LLANOS, 2010; ZHANG; LIU, 2008), nanocubos (IN; VAUGHN; SCHAAK, 2012), entre outras. Para a síntese solgel realizada, os resultados foram compatíveis com o relatado na literatura, uma vez que o material não calcinado apresentou a formação de aglomerados de centenas de nanômetros. Já para o material calcinado foi possível obter estruturas de anéis de $\mathrm{TiO}_{2}$ na ordem de algumas centenas de nanômetros de diâmetro. Esse 
resultado mostra o potencial dessa metodologia para futuras tentativas de obtenção de uma morfologia diferente para o $\mathrm{TiO}_{2}$ e mais estudos precisam ser realizados visando a elucidação dessa morfologia.

$\mathrm{Na}$ Figura 3(b) é possível perceber uma maior uniformidade de distribuição de tamanho de partículas, indicando que a temperatura de 450 ${ }^{\circ} \mathrm{C}$ já é suficiente para obtenção de um material tamanho homogêneo, esse comportamento é mantido enquanto a temperatura de calcinação foi $550^{\circ} \mathrm{C}$. Conforme a Figura 3(c), pode-se perceber a presença de aglomerados e a manutenção dos anéis obtidos em temperatura inferior.

\section{CONCLUSÃO}

As metodologias sol-gel e solvotérmica assistida por microondas foram eficientes para a obtenção de nanopartículas de $\mathrm{TiO}_{2}$, onde se observou a formação da fase anatase. Pôde-se atingir a estrutura de $\mathrm{TiO} 2$ para o material sintetizado pela síntese sol-gel e a cristalinidade das amostras apresentou influência direta com o tempo de calcinação, onde quanto maior o tempo, maior a cristalinidade, para a metodologia sol-gel. Entretanto em aplicacóes onde esse fator não é importante a calcinação não se faz necessária. Já a metodologia solvotérmica apresentou aumento no tamanho dos cristais quando utilizadas maiores temperaturas de síntese. Quanto a morfologia, observou-se a presença de grandes aglomerados para o material que seguiu a metodologia sol-gel e não foi calcinado. Já para o material calcinado gerou-se aglomerados e uma estrutura anelar de óxido de titânio. Análises de microscopia eletrônica de transmissão, bem como análise de potencial Zeta se fazem necessárias para maiores informaçóes a respeito dos aglomerados das nanopartículas.

\section{AGRADECIMENTOS}

Os autores agradecem ao Laboratório de Caracterização Estrutural dos Materiais (LCEM) da Universidade Federal do Rio Grande do Norte e seus técnicos, pelas análises de DRX e MEV-FEG e à Coordenação de Aperfeiçoamento de Pessoal de Ensino Superior (CAPES) pelo suporte financeiro.

\section{LITERATURA CITADA}

ARCONADA, N. et al. Synthesis and photocatalytic properties of dense and porous $\mathrm{TiO} 2$-anatase thin films prepared by solgel. Applied Catalysis B: Environmental, v. 86, n. 1-2, p. 1-7, 2009.

BEHNAJADY, M. A. et al. Sol-Gel Low-temperature Synthesis of Stable Anatase-type TiO2 Nanoparticles Under Different Conditions and its Photocatalytic Activity. Photochemistry and Photobiology, v. 87, p. 1002-1008, 2011.

BESSEKHOUAD, Y.; ROBERT, D.; WEBER, J. V. Preparation of $\mathrm{TiO} 2$ nanoparticles by Sol-Gel route. International Journal of Photoenergy, v. 5, n. January, p. 2-7, 2003.

BIAN, Z. et al. Solvothermal synthesis of well-defined $\mathrm{TiO} 2$ mesoporous nanotubes with enhanced photocatalytic activity. Chemical Communications, v. 46, n. 44, p. 8451-8453, 2010.

CRAWFORD, G. A. et al. Microstructure and deformation behavior of biocompatible $\mathrm{TiO} 2$ nanotubes on titanium substrate. Acta Biomaterialia, v. 3, n. 3 SPEC. ISS., p. 359-367, 2007.

DI PAOLA, A.; BELLARDITA, M.; PALMISANO, L. Brookite, the Least Known TiO2 Photocatalyst. [s.l: s.n.]. v. 3

FAN, Z. et al. Solvothermal synthesis of hierarchical TiO 2 nanostructures with tunable morphology and enhanced photocatalytic activity. Applied Surface Science, v. 360, p. 298 305, 2016.

GUPTA, S.; TRIPATHI, M. A review on the synthesis of TiO2 nanoparticles by solution route. Open Chemistry, v. 10, n. 2, 2012 .

HAIDER, A. J. et al. Exploring potential Environmental applications of TiO2 Nanoparticles. Energy Procedia, v. 119, p. 332-345, 2017.

HANAOR, D. A. H.; SORRELL, C. C. Review of the anatase to rutile phase transformation. Journal of Materials Science, v. 46, n. 4, p. 855-874, 2011.

HOU, X. G. et al. Preparation and studies of photocatalytic silver-loaded TiO2films by hybrid sol-gel method. Chemical Engineering Journal, v. 146, n. 1, p. 42-48, 2009.

IN, S. IL; VAUGHN, D. D.; SCHAAK, R. E. Hybrid CuO$\mathrm{TiO} 2$ hollow nanocubes for photocatalytic conversion of $\mathrm{CO} 2$ into methane under solar irradiation. Angewandte Chemie International Edition, v. 51, n. 16, p. 3915-3918, 2012.

KIM, D. S.; KWAK, S. Y. The hydrothermal synthesis of mesoporous $\mathrm{TiO} 2$ with high crystallinity, thermal stability, large surface area, and enhanced photocatalytic activity. Applied Catalysis A: General, v. 323, p. 110-118, 2007.

LÓPEZ, R.; GÓMEZ, R.; LLANOS, M. E. Photophysical and photocatalytic properties of nanosized copper-doped titania solgel catalysts. Catalysis Today, v. 148, n. 1-2, p. 103-108, 2010. 
MACWAN, D. P.; DAVE, P. N.; CHATURVEDI, S. A review on nano-TiO2sol-gel type syntheses and its applications. Journal of Materials Science, v. 46, n. 11, p. 3669-3686, 2011.

SUPPHASRIRONGJAROEN, P. et al. Effect of quenching medium on photocatalytic activity of nano-TiO2 prepared by solvothermal method. Chemical Engineering Journal, v. 138, n. 1-3, p. 622-627, 2008.

TSUCHIYA, H. et al. Nanotube Layers With Metallic Nanoparticles. Journal of Physics: Conference Series, v. 165, p. $012037,2009$.

WANG, H. et al. Single-Crystalline Rutile TiO2 Hollow Spheres: Room-Temperature Synthesis, Tailored Visible-Light-Extinction and Effective Scattering Layer for Quantum-Dot-Sensitized Solar Cells. Journal of the American Chemical Society, p. 19102$19109,2011$.

WANG, Y. et al. Review of the progress in preparing nano TiO: An important environmental engineering material. Journal of Environmental Sciences (China), v. 26, n. 11, p. 2139-2177, 2014.

YOU, Y. et al. Preparation of continuous TiO2fibers by sol-gel method and its photocatalytic degradation on formaldehyde. Applied Surface Science, v. 258, n. 8, p. 3469-3474, 2012.

ZHANG, X.; LIU, Q. Preparation and characterization of titania photocatalyst co-doped with boron, nickel, and cerium. Materials Letters, v. 62, n. 17-18, p. 2589-2592, 2008.

DEVIKALA, S. et al. AC conductivity studies of PMMA/TiO2 composites, Materials Today: Proceedings, Volume 5, Issue 2, Part 3, 2018, Pages 8678-8682,

ZU, G. et al. Highly efficient mass determination of $\mathrm{TiO} 2$ nanotube arrays and its application in lithium-ion batteries, Sustainable Materials and Technologies, Volume 18, 2018,

ANDRADE NETO, N. F. et al. Increase of antimicrobial and photocatalytic properties of silver-doped $\mathrm{PbS}$ obtained by sonochemical method, Journal of Materials Science: Materials in Electronics, 2018, 10.1007/s10854-018-0031-z. 